\title{
Microvascular Dysfunction Related to Progressive Left Ventricular Remodeling due to Chronic Occlusion of the Left Anterior Descending Artery in an Adult Porcine Heart
}

\author{
Shin Yajima, ${ }^{1} \mathrm{MD}$, Shigeru Miyagawa, ${ }^{1} \mathrm{MD}$, Satsuki Fukushima, ${ }^{1} \mathrm{MD}$, Kayako Isohashi, ${ }^{2} \mathrm{MD}$, \\ Tadashi Watabe, ${ }^{2} \mathrm{MD}$, Hayato Ikeda, ${ }^{2} \mathrm{PhD}$, Genki Horitsugi, ${ }^{2} \mathrm{PhD}$, Akima Harada, ${ }^{1} \mathrm{BSc}$, \\ Ryoto Sakaniwa, ${ }^{3} \mathrm{PhD}$, Jun Hatazawa, ${ }^{2} \mathrm{MD}$ and Yoshiki Sawa, ${ }^{1} \mathrm{MD}$
}

\begin{abstract}
Summary
Occlusion of a major coronary artery induces myocardial infarction (MI), leading to left ventricle (LV) remodeling due to progressive microvasculature dysfunction. Irreversible impairment in microvascular function has been suggested to extend from the infarcted region into the infarct-border or remote regions, depending on the time to revascularization. Our aim was to determine whether the occlusion of a major coronary artery induces microvascular dysfunction in the adjacent area perfused by intact coronary arteries using a porcine model for chronic total occlusion of the left anterior descending artery (LAD). MI was induced via an ameroid constrictor ring around the LAD in adult Göttingen pigs (Sus scrofa domesticus, $n=5$ ). Age-matched normal pigs were treated as controls $(n=3)$. Cardiac magnetic resonance showed reduced systolic regional wall motion in the left circumflex $(\mathrm{LCx})$ and right coronary artery (RCA) territories, with a progressively worsening motion in the infarction-adjacent area over an eight-week period. On ${ }^{13} \mathrm{~N}$-ammonia positron emission tomography (PET), myocardial blood flow (MBF) during hyperemia was significantly greater in the LCx and RCA territories (particularly in the infarction-adjacent area) compared to that in the LAD territory at four weeks after infarct induction. Subsequently, the flow significantly decreased, approaching that in the LAD territory at eight weeks after infarct induction. Fluoroscopy-guided pressure-wire studies showed significantly higher microvascular resistance in the LCx area at eight weeks compared to that in controls. Electron microscopy showed endothelium swelling and microvasculature disruption in areas adjacent to the LCx and RCA territories. Anterior MI caused coronary microvascular dysfunction in the adjacent area, associated with a reduced MBF and regional wall motion.
\end{abstract}

(Int Heart J 2019; 60: 715-727)

Key words: Myocardial infarction, Regional cardiac wall motion, Myocardial blood flow, Microvascular resistance

$\mathrm{M}$ yocardial infarction (MI) in the territory of a major coronary artery branch, such as the left anterior descending artery (LAD), represents a critical pathology of the adult heart. ${ }^{1,2)}$ Although revascularization therapy is an established treatment for this pathology, its effectiveness is largely dependent on completeness $^{3)}$ or time to revascularization. ${ }^{4)}$ Delayed or a lack of revascularization is associated with progressive remodeling of the left ventricle (LV), leading to advanced congestive cardiac failure, despite other coronary arteries being intact. ${ }^{5,6)}$ Recently, irreversible impairments in the microvascular function have been suggested to extend from the infarcted region into the infarct-border or remote regions, depending on the time to revascularization; these impairments are associated with progression in LV remod- eling. ${ }^{7}$

Here, we hypothesize that MI in the LAD territory might induce microvascular dysfunction in the infarctborder region, resulting in an impaired regional function. In order to test this hypothesis, we used a porcine model for chronic total occlusion of the LAD and comprehensively investigated the regional wall motion, blood flow, and pathologies using the latest imaging modalities, including cardiac magnetic resonance imaging (CMR), ${ }^{13} \mathrm{~N}-$ ammonia positron emission tomography (PET), a fluoroscopy-guided pressure-wire study, and transmission electron microscopy (TEM).

From the ${ }^{1}$ Department of Cardiovascular Surgery, Osaka University Graduate School of Medicine, Osaka, Japan, ${ }^{2}$ Department of Nuclear Medicine and Tracer Kinetics, Osaka University Graduate School of Medicine, Osaka, Japan and ${ }^{3}$ Public Health, Osaka University Graduate School of Medicine, Osaka, Japan.

This work was supported by Grants-in-Aid for Scientific Research (KAKENHI) [grant number 15K10214].

Address for correspondence: Yoshiki Sawa, MD, Department of Cardiovascular Surgery, Osaka University Graduate School of Medicine, 2-2 Yamadaoka, Suita, Osaka 565-0871, Japan. E-mail: s-yajima@surg1.med.osaka-u.ac.jp

Received for publication June 2, 2018. Revised and accepted October 23, 2018

Released in advance online on J-STAGE May 17, 2019.

doi: 10.1536/ihj.18-346

All rights reserved by the International Heart Journal Association. 
A

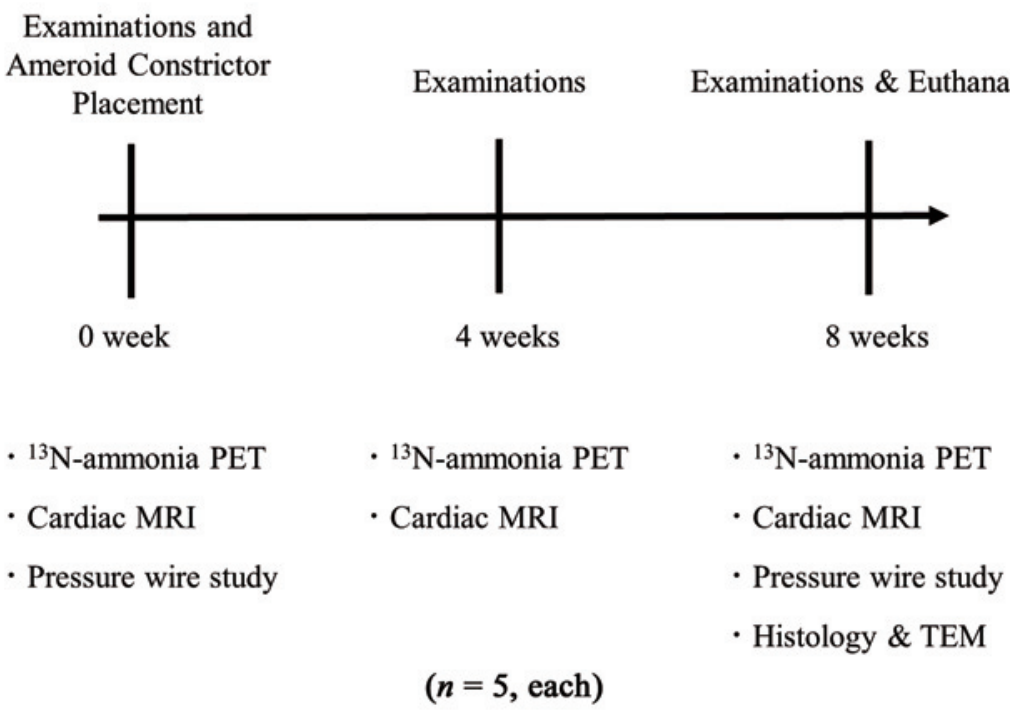

B
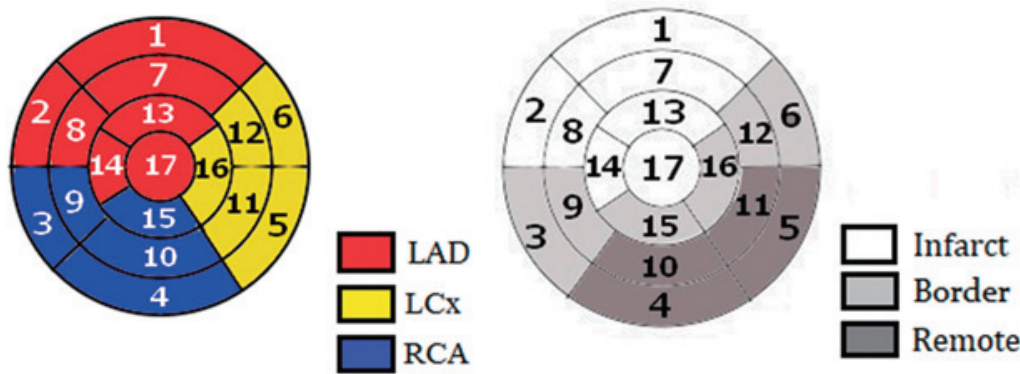

Figure 1. A: Study protocol. B: Definition of the myocardial areas, according to the domination of the coronary artery (left) and the area of the ischemic insult (right). PET indicates positron emission tomography; MRI, magnetic resonance imaging; TEM, transmission electron microscopy; LAD, left anterior descending artery; LCx, left circumflex artery; and RCA, right coronary artery.

\section{Methods}

The experimental procedures were approved by the institutional ethics committee. Animal care was conducted in compliance with the Principles of Laboratory Animal Care formulated by the National Society for Medical Research and the Guide for the Care and Use of Laboratory Animals (8th Edition, 2011) prepared by the Institute of Animal Resources and published by the National Institutes of Health.

Study protocol: Five male Göttingen minipigs (Ellegaard, Dalmose, Denmark), weighing 20-25 kg, were anesthetized with an intravenous administration of ketamine (6 $\mathrm{mg} / \mathrm{kg})$ and sodium pentobarbital $(10 \mathrm{mg} / \mathrm{kg})$ for endotracheal intubation; anesthesia was maintained with inhaled sevoflurane $(1.5-2 \%)$, and mechanical ventilation was performed. Vital signs were monitored continuously to confirm the depth of anesthesia. A chronic MI model was generated by placing an ameroid constrictor ring (MRI-
2.50-TI; Research Instruments NW Inc., Lebanon, OR, USA), with an internal diameter of $4.0 \mathrm{~mm}$, around the proximal portion of the LAD through the left fourth intercostal space. ${ }^{89)}$ The skin incision was then closed in layers, and the minipigs were cared for during recovery in temperature-controlled individual cages. General anesthesia was again induced at four and eight weeks after ameroid constrictor placement for electrocardiogram(ECG-) gated CMR imaging $(n=5)^{10)}$ and ${ }^{13} \mathrm{~N}$-ammonia PET $(n=5)$ studies. At eight weeks, a fluoroscopy-guided pressure-wire study was performed after the CMR study. All chronic MI model pigs and three other normal (control) pigs were euthanized at eight weeks via an intraperitoneal injection of pentobarbital $(200 \mathrm{mg} / \mathrm{kg})$ to provide immediate pain relief and an intravenous injection of a potassium solution $(20 \mathrm{mEq})$. The cardiac tissue was then harvested for histological studies (Figure 1A).

Definition of myocardial areas: A cardiac segmental analysis was performed in accordance with the American 
Heart Association 17-segment model. ${ }^{11)}$ These 17 segments were categorized into three territories [LAD, left circumflex artery (LCx), and right coronary artery (RCA)], and the segments were categorized into infarct, border, and remote areas, as shown in Figure 1B. Segment 17 was excluded from the analysis because of a lack of methodological authenticity.

CMR data acquisition and feature-tracking analysis: Horizontal long-axis and short-axis images from the atrioventricular ring to the LV apex were acquired on a 1.5 T scanner (Signa EXCITE XI TwinSpeed; GE Medical Systems, Milwaukee, WI, USA), producing steadystate free-precession images of the basal, midventricular, and apical LV levels in the short-axis views, as well as apical four-chamber, two-chamber, and long-axis views. The images were analyzed using commercially available feature-tracking software (2D CPA MR; TomTec Imaging Systems, Unterschleissheim, Germany), with a vectorbased analytical tool based on a hierarchical algorithm that was previously validated in clinical studies. ${ }^{12)}$ The LV endocardial border at the end-diastolic frame was manually drawn on a single frame by an expert reader for each of the three short-axis and long-axis plane images. The software then automatically propagated the contour and followed its features throughout the cardiac cycle to determine the longitudinal strain and strain rate of the 17 segments, as well as the circumferential strain and strain rate of 16 segments, excluding segment 17 . The global strain and strain rate were determined by averaging the peak values of the segmental longitudinal and circumferential strains and strain rates, respectively.

${ }^{13} \mathrm{~N}$-ammonia PET protocol and data analysis: ${ }^{13} \mathrm{~N}$ ammonia myocardial perfusion PET was performed using a Headtome-V/SET 2400 W PET System (Shimadzu, Kyoto, Japan), with the animals in the left lateral decubitus position on the scanner bed and under general anesthesia. 47 slices were obtained, with center-to-center distances of $3.125 \mathrm{~mm}$ and an intrinsic spatial resolution of $4.0 \mathrm{~mm}$ in-plane and $4.3 \mathrm{~mm}$ full-width at half-maximum axially. At rest, the minipigs were injected with $192 \pm 24$ $\mathrm{MBq}$ of ${ }^{13} \mathrm{~N}$-ammonia via an ear vein, followed by a 10 $\mathrm{mL}$ saline flush. Dynamic imaging was started simultaneously with the injection and continued for 10 minutes. 15 minutes later, an adenosine stress test was performed, using an infusion of adenosine at $180 \mu \mathrm{g} / \mathrm{kg} / \mathrm{minute}$ for 10 minutes to induce a hyperemic state. After 5 minutes of infusion, $380 \pm 88 \mathrm{MBq}$ of ${ }^{13} \mathrm{~N}$-ammonia was injected, and stress dynamic imaging data were acquired for 10 minutes in the two-dimensional mode. 25 dynamic frames were reconstructed $(6 \times 10$ seconds, $6 \times 20$ seconds, $12 \times$ 30 seconds, and $1 \times 60$ seconds, for a total of $10 \mathrm{~min}-$ utes), using a $128 \times 128$ matrix and filtered backprojection. All emission data were corrected for tissue attenuation by means of a transmission scan with an external source of ${ }^{68} \mathrm{Ge}^{68} \mathrm{Ga}$. Continuous ECG monitoring was conducted, and the heart rate, blood pressure, and 12-lead ECG data were recorded.

Using ${ }^{13} \mathrm{~N}$-ammonia PET images, myocardial blood flow (MBF) values were estimated using the commercially available PMOD software package (version 3.4; PMOD Technologies, Ltd., Zurich, Switzerland). Reconstructed images were resliced in the short axis and vertical and horizontal long-axis orientations. Regions of interest in the LV myocardium were automatically generated and minimally modified by an experienced reader (as necessary to best match the cardiac anatomy and avoid contamination from extracardiac activity). Myocardial and blood-pool time-activity curves, generated from the dynamic frames, were fitted using the tracer kinetic model. We adopted the DeGrado one-compartment model, which assumes that there is no metabolic trapping. ${ }^{13)}$ Rest and stress MBFs were estimated for each segment, territory, and area.

Fluoroscopy-guided coronary angiography and pressure-wire study: Fluoroscopy-guided selective coronary angiography was performed using an injection of iopamidol (Isovue 370; Bracco Diagnostics, Monroe Township, NJ) via a catheter (VISTA BRITE TIP; Cordis, Miami, FL, USA) inserted into the right femoral artery, with the minipigs in the supine position and under general anesthesia. Subsequently, a fluoroscopy-guided pressure wire (Radi Medical Systems, Uppsala, Sweden) was inserted to assess the myocardial microvascular resistance in the LCx and RCA territories, using the method described by Fearon et al. ${ }^{14)}$ Briefly, the index of microvascular resistance (IMR) was determined under resting and hyperemic conditions. The mean aortic and distal coronary pressures were recorded during peak hyperemia. Coronary flow reserve (CFR) was calculated from the ratio of hyperemic to resting coronary flow recorded from the flow probe.

Histology and immunohistolabeling: Modulations in angiogenic, myocyte morphologic, and fibrotic profiles in the infarct, peri-infarct, and remote areas were histologically assessed at eight weeks after ameroid placement, with corresponding areas assessed in normal controls. Excised heart samples were fixed with $10 \%$ buffered formalin in paraffin-embedded sections. $5 \mu \mathrm{m}$ slices of the paraffin-embedded sections were stained with periodic acid-Schiff (PAS) or Sirius Red and evaluated under an optical microscope (Leica, Wetzlar, Germany). Quantitative morphometric analyses were performed using MetaMorph software (Molecular Devices, Sunnyvale, CA, USA). Sirius Red staining was performed to assess myocardial fibrosis in the peri-infarct region. The fibrotic region was calculated as the percentage of the myocardial area. Data were obtained from five individual views of each heart. PAS staining was performed to examine the degree of cardiomyocyte hypertrophy. Myocyte size was determined by drawing point-to-point perpendicular lines across the cross-sectional area of the cell at the level of the nucleus. The results are expressed as the average diameter of myocytes randomly selected from 10 fields of each ventricle. ${ }^{15)}$ In addition, the paraffin sections were labeled for anti-CD31 antibodies (Abcam, Cambridge, UK) and visualized using LSABTM kits (Dako, Glostrup, Denmark). Vessel density was quantified as the number of $\mathrm{CD}$ 31-positive vessels per millimeter squared. ${ }^{16)}$

Transmission electron microscopy (TEM): Porcine hearts were harvested immediately following euthanasia. The LV tissue was fixed overnight at $4^{\circ} \mathrm{C}$ using $1 / 2$ strength Karnovsky's Fixative (primary fixation), followed by $2 \%$ osmium tetroxide at $4^{\circ} \mathrm{C}$ for 2 hours (postfixa- 
tion). The fixed myocardium was dehydrated using ethanol and embedded with epoxy resin. Ultrathin sections of the infarct, border, and remote regions were prepared using a Leica EM UC7 ultramicrotome (90-120 nm thick) and stained with uranyl acetate and lead citrate on a grid for TEM (H-7650; Hitachi, Tokyo, Japan). ${ }^{17)}$

Statistical analysis: JMP software (JMP 12; SAS Institute, Inc., Cary, NC, USA) was used for statistical analysis. Continuous values are presented as the mean \pm standard deviation. The analyses were performed using nonparametric methods because the sample sizes were too small to determine a normal or skewed distribution. Between-group differences were compared using KruskalWallis analysis with a Mann-Whitney $U$ test ad hoc analysis. $P$-values less than 0.05 indicated statistical significance.

\section{Results}

Reduced wall motion in the peri-infarct areas: CMR imaging revealed substantially thinner anteroseptal LV walls (from the base to the apex) at four and eight weeks after ameroid placement compared to that in the controls (Figure 2). In addition, the LV end-diastolic volume (LVEDV) and LV end-systolic volume (LVESV) were significantly larger, whereas the LV ejection fraction (LVEF) was significantly smaller, at four weeks $(46 \pm 8 \mathrm{~mL}, 30 \pm$ $7 \mathrm{~mL}$, and $34 \% \pm 3 \%$, respectively) and eight weeks (54 $\pm 6 \mathrm{~mL}, 35 \pm 4 \mathrm{~mL}$, and $33 \% \pm 8 \%$, respectively) compared to that in the controls $(25 \pm 5 \mathrm{~mL}, 11 \pm 2 \mathrm{~mL}$, and $52 \% \pm 2 \%$, respectively; $P<0.01)$. The LVEDV, LVESV, and LVEF at four and eight weeks after ameroid placement were not significantly different.

The longitudinal and circumferential strains, stratified by coronary artery domination and the area of the ischemic insult, are shown in Figure 3. The global longitudinal strain was significantly smaller at four and eight weeks compared to that in the controls. The longitudinal strains in the LAD and LCx territories, but not in the RCA territory, were significantly smaller at four and eight weeks compared to that in the controls. Similarly, the longitudinal strains in the infarct and border areas, but not in the remote area, were significantly smaller at four and eight weeks compared to that in the controls. Furthermore, the global and territorial longitudinal strains at four and eight weeks after ameroid placement were not significantly different. The trends observed in the circumferential strain were the same as those observed in the longitudinal strain.

The longitudinal and circumferential strain rates, stratified by coronary artery domination and the area of the ischemic insult, are shown in Figure 4. The results for the longitudinal and circumferential strain rates showed slightly different trends from those for the longitudinal and circumferential strains. The longitudinal and circumferential strain rates in the global and infarct/border areas, but not in the remote area, were significantly smaller at four and eight weeks after ameroid placement compared to that in the controls. However, the longitudinal and circumferential strain rates in the LAD, LCx, and RCA territories were significantly smaller at four and eight weeks after ameroid placement compared to that in the controls. Time-dependent reduction of $\mathrm{MBF}$ in the peri-infarct area: ${ }^{13} \mathrm{~N}$-ammonia myocardial perfusion PET revealed significantly reduced global $\mathrm{MBF}$ at eight weeks after ameroid placement $(1.1 \pm 0.2 \mathrm{~mL} /$ minute $/ \mathrm{g})$ compared to that in the controls $(1.8 \pm 0.5 \mathrm{~mL} /$ minute $/ \mathrm{g} ; P<0.01$; Figure 5). Global MBF at four weeks after ameroid placement $(1.4 \pm 0.1 \mathrm{~mL} / \mathrm{minute} / \mathrm{g})$ was not significantly different from that in the controls.

The MBF in the LAD territory was significantly reduced at four and eight weeks compared to that in the controls, without a significant difference between the values at four and eight weeks. At four weeks after ameroid placement, the MBF in the LCx and RCA territories was significantly greater than that in the LAD territory but was not significantly different from that in the controls. Interestingly, the MBF in the LCx and RCA territories at eight weeks was significantly reduced compared to that in the controls. At four weeks, the MBF in the border and remote areas was significantly greater compared to that in the infarct area but was not significantly different from that in the controls. Interestingly, at eight weeks after ameroid placement, the MBF in the border area, but not in the remote area, was significantly reduced compared to that in the controls.

Functional impairment of macro- and microvasculature in the LCx territory: At eight weeks, the proximal LAD was completely occluded in all experimental animals, with collateral arteries from the LCx branches to the distal LAD and septal branches. The RCA was intact, with minimal collateral arteries from its branches to the LAD territory (Figure 6).

The CFR in the LCx territory, but not the RCA territory, was significantly smaller at eight weeks compared to that in the controls $(P<0.05)$. Similarly, the IMR in the LCx territory, but not in the RCA territory, was significantly greater at eight weeks compared to that in the controls $(P<0.05)$.

Pathological remodeling of the peri-infarct myocardium: Histological analysis revealed that CD31-positive capillaries were markedly less dense in the infarct and peri-infarct areas than in the remote area (Figure 7). In addition, myocyte size in the peri-infarct area and the number of fibrous components were greater than in the remote area. The capillary density, myocyte size, and the number of fibrous components in the remote area did not significantly differ from those in the controls.

Narrowing capillaries and disarraying myofibrils in the peri-infarct myocardium: TEM at eight weeks revealed that, in the infarct area, capillaries were obstructed and surrounded by fibrotic tissue and myofibrils were disturbed in orientation and had degenerated to vacuolization (Figure 8). In contrast, capillaries in the peri-infarct area were narrowed because of the endothelial swelling and were filled with serum component, and the Z-bands were disarrayed in the myofibrils. The remote area had narrowed capillaries, with frequently observed endothelial swelling and few damaged myofibrils. 


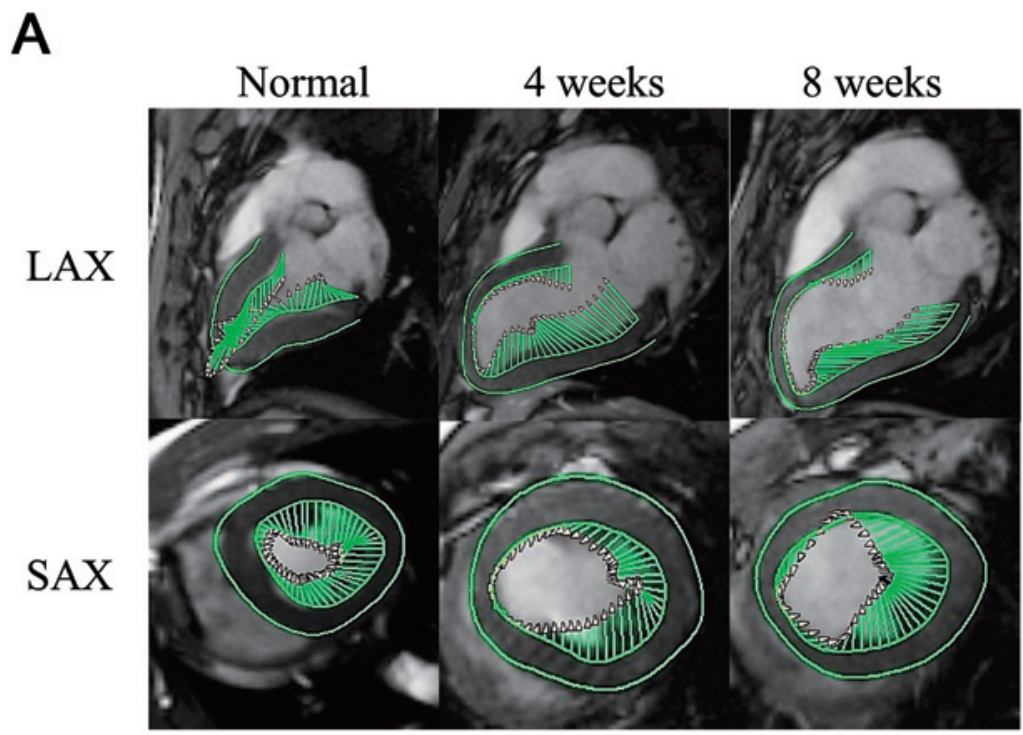

B

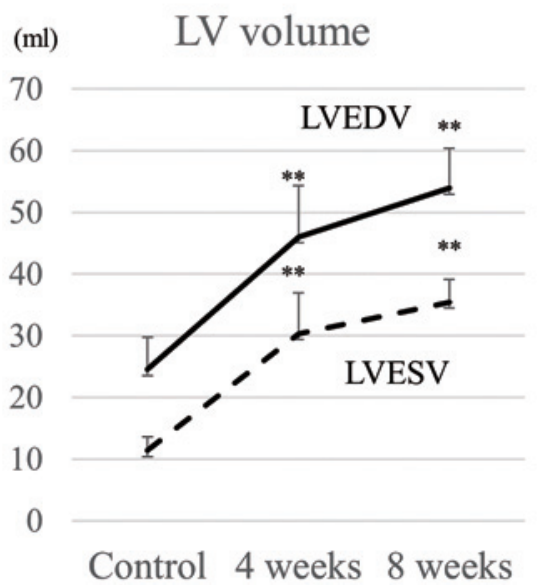

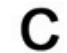

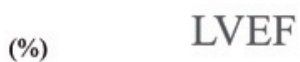

60

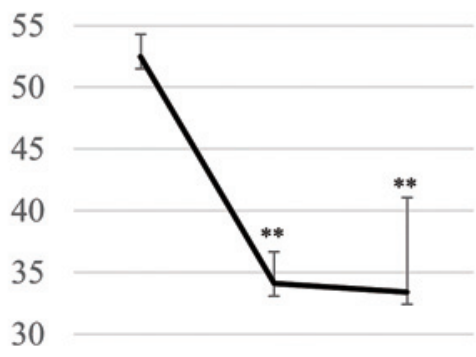

25

\section{Control 4 weeks 8 weeks}

Figure 2. Representative cardiac images of the long (top) and short (bottom) axial views at the end-systolic phase (A). Changes in the LV volume (B) and LVEF (C) at four and eight weeks after MI relative to those in the controls $(n=5)$. $* * P<0.01$ compared to controls, as assessed using the Mann-Whitney $U$ test. LAX indicates long axis; SAX, short axis; LVEDV, left ventricular end-diastolic volume; LVESV, left ventricular end-systolic volume; and LVEF, left ventricular ejection fraction.

\section{Discussion}

Principal findings: In the present study, total occlusion of the LAD (via the placement of an ameroid constrictor around the LAD) in an adult porcine heart induced the development of collateral arteries from the LCx branches and was associated with a substantial impairment in global LV function over an eight-week period. Ameroid placement around the LAD also induced an impairment in regional LV function, including the longitudinal and circumferential strains and strain rates, predominantly in the
LAD/infarct areas, less substantially (but significantly) in the LCx/border areas, and even in the RCA area. In addition, ameroid placement around the LAD reduced MBF in all territories, predominantly in the LAD/infarct areas, which was associated with significantly elevated IMR in the LCx area. Pathologically, a reduction in microvascular density, hypertrophy of the cardiac myocytes, and an accumulation of fiber contents were evident in the LAD/infarct areas and less predominantly, but significantly, in the LCx/border areas. In addition, capillaries in the border area were narrowed because of the swelling of the endo- 

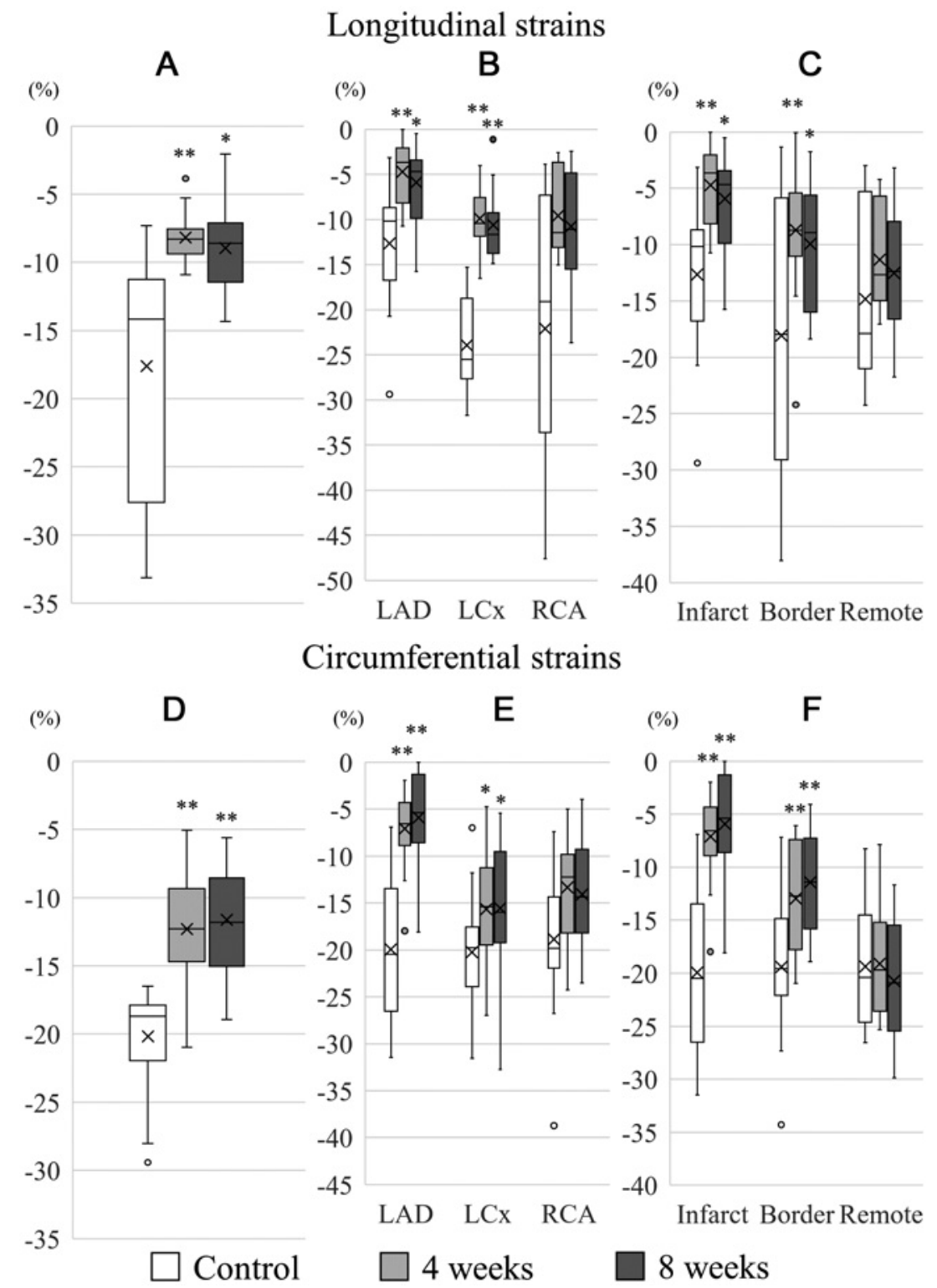

Figure 3. Longitudinal (top) and circumferential (bottom) strains. Global (A) and regional longitudinal strains, stratified by the domination of the coronary artery $(\mathbf{B})$ and the area of the ischemic insult (C). Global (D) and regional circumferential strains, stratified by the domination of the coronary artery $(\mathbf{E})$ and the area of the ischemic insult $(\mathbf{F})(n=5)$. The upper and lower borders of the box represent the upper and lower quartiles, respectively. The middle horizontal line represents the median. The upper and lower whiskers represent the maximum and minimum values of nonoutliers, respectively. The extra dots represent outliers. $* P<0.05$, $* * P<0.01$ compared to controls, as assessed using Kruskal-Wallis analysis and the Mann-Whitney $U$ test. LAD indicates left anterior descending artery; LCx, left circumflex artery; and RCA, right coronary artery.

thelium, and myofibril Z-bands in the border area were disarrayed.

The results of the present study support the concept that MI-associated microvascular dysfunction extends into the area adjacent to the infarct. In addition, the responsible mechanisms could be speculated upon, at least in part, as follows. Occlusion of the proximal LAD induces the development of collateral arteries in the peri-infarct area, predominantly from the LCx territory, whereas myocyte hypertrophy and fibrous tissue accumulation in the periinfarct area are induced in association with functional impairment in this area. The functionality and/or maturity of the microvascular network formed by resident and new vessels in the peri-infarct area may determine the oxygen supply in this area and, consequently, the functional fate of this area (e.g., complete recovery or progressive deterioration). In addition, the functionality and/or maturity of the microvascular network in the peri-infarct area would 


\section{Longitudinal strain rate}
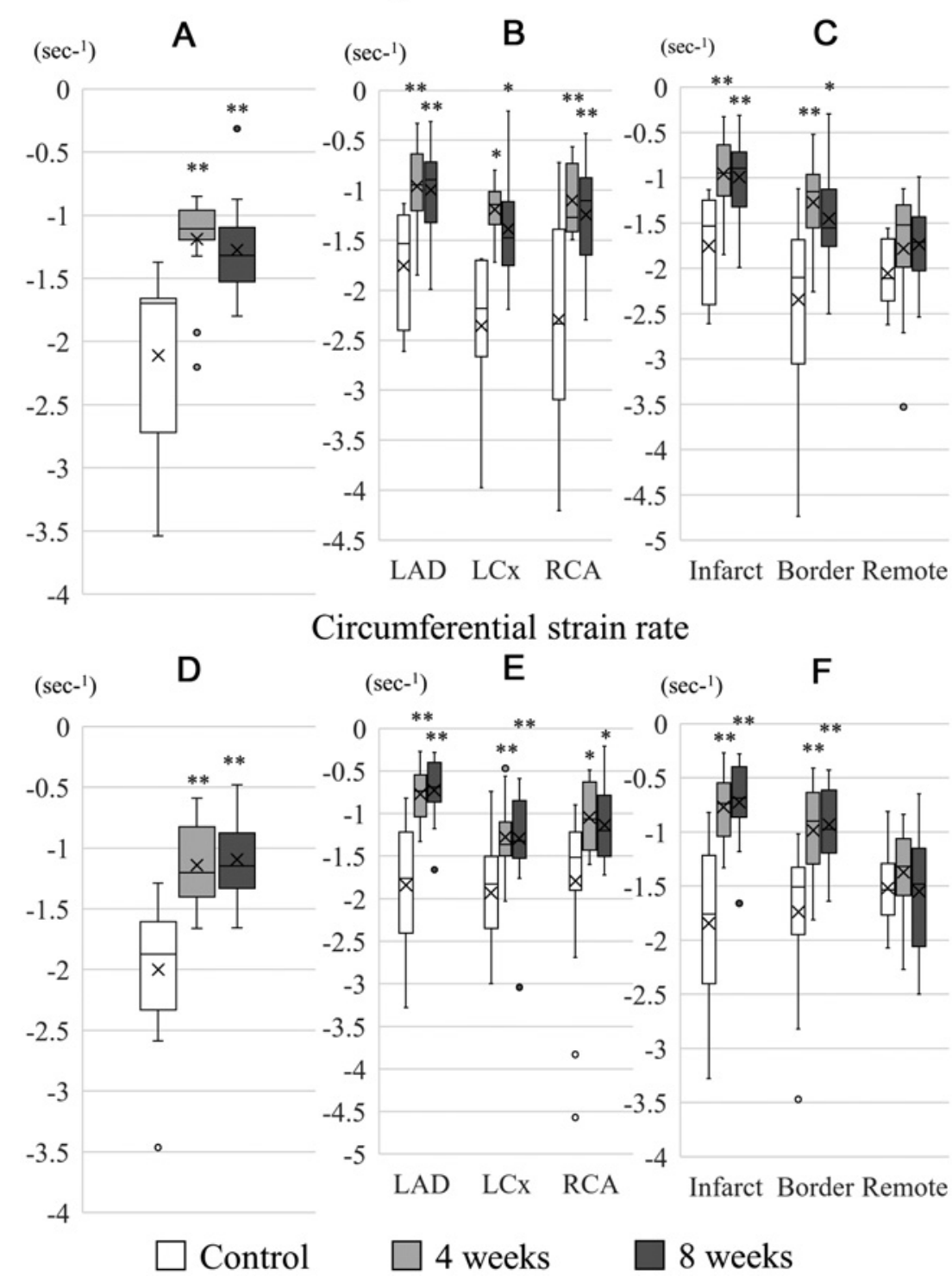

Figure 4. Longitudinal (top) and circumferential (bottom) strain rates. Global (A) and regional longitudinal strain rates, stratified by the domination of the coronary artery $(\mathbf{B})$ and the area of the ischemic insult (C). Global (D) and regional circumferential strain rates, stratified by the domination of the coronary artery $(\mathbf{E})$ and the area of the ischemic insult $(\mathbf{F})(n=5)$. The upper and lower borders of the box represent the upper and lower quartiles, respectively. The middle horizontal line represents the median. The upper and lower whiskers represent the maximum and minimum values of nonoutliers, respectively. The extra dots represent outliers. $* P<0.05$, $* * P<0.01$ compared to controls, as assessed using Kruskal-Wallis analysis and the Mann-Whitney $U$ test. LAD indicates left anterior descending artery; LCx, left circumflex artery; and RCA, right coronary artery.

be determined by the magnitude and nature of the ischemic insult, the nature of the native cardiac tissue, and/or post-MI treatments. ${ }^{6}$

Applicability of porcine model to human MI: In the present porcine MI model, the LAD underwent slow constriction without reperfusion; the coronary artery was intact, without pathological plaque formation; and no postMI drug therapy was administered. Anatomical and physiological differences between the human and porcine heart may cause the present findings to not be completely translatable to the human adult heart with MI in the LAD territory. However, we consider the principal concept of the present findings (i.e., that microvascular dysfunction extends to the area adjacent to the infarct) to be relevant to human pathology for the following reasons. First, the ameroid-induced MI porcine model is the standard model for chronic MI and has been used in numerous preclinical studies for the development of drugs or devices. ${ }^{18,19)}$ More- 
A

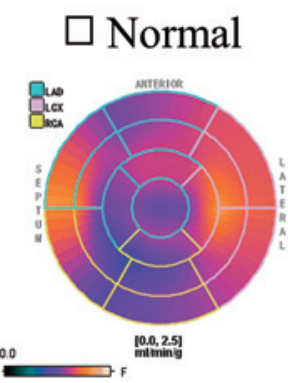

4 weeks
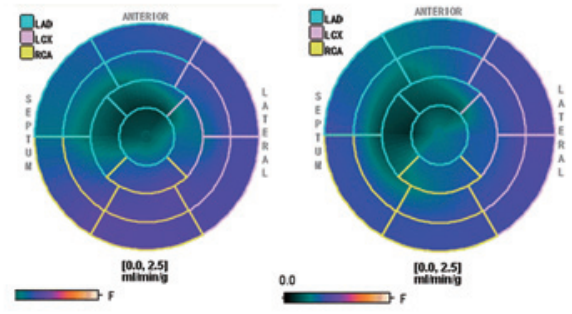

B $(\mathrm{mL} /$ minute/g)

Global MBF
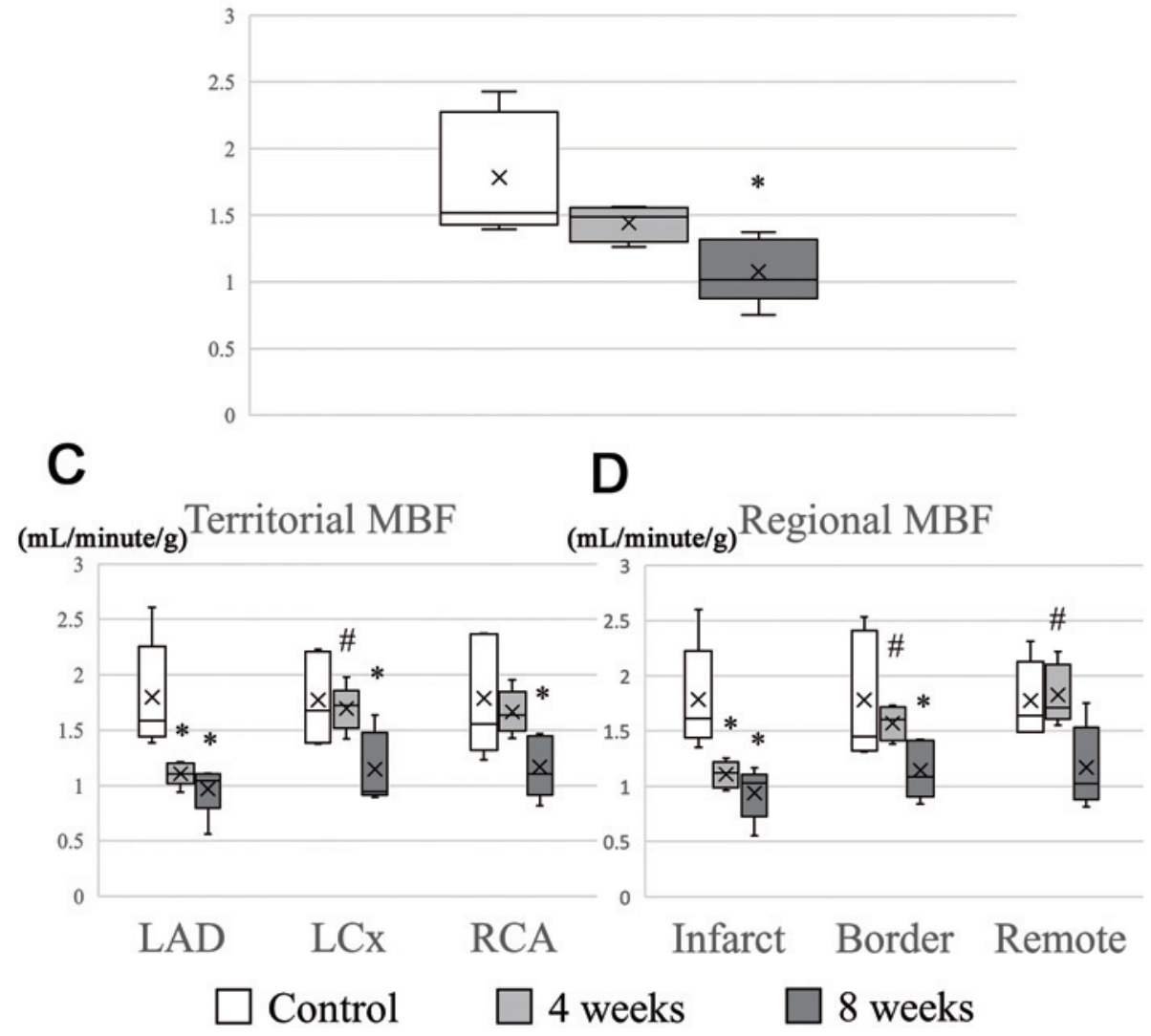

Figure 5. Representative bullseye of the hyperemic $\operatorname{MBF}(\mathbf{A})$ and global hyperemic $\mathrm{MBF}(\mathbf{B})$ in the controls and at four and eight weeks after MI. Hyperemic MBF in the LAD, LCx, and RCA territories (C). Hyperemic MBF in the infarct, border, and remote regions $(\mathbf{D})(n=5)$. The upper and lower borders of the box represent the upper and lower quartiles, respectively. The middle horizontal line represents the median. The upper and lower whiskers represent the maximum and minimum values of nonoutliers, respectively. The extra dots represent outliers. ${ }^{*} P<0.05$ compared to controls, ${ }^{\#} P<0.05$ compared to the LAD territory value at four weeks, as assessed using Kruskal-Wallis analysis and the Mann-Whitney $U$ test. MBF indicates myocardial blood flow; LAD, left anterior descending artery; LCx, left circumflex artery; and RCA, right coronary artery.

over, although this model enhances the development of collateral arteries in the infarct and peri-infarct areas by slowly inducing myocardial ischemia, the dysfunction of the microvascular network was shown to extend to the peri-infarct area. Microvascular dysfunction in the periinfarct area would be expected to be more affected in the adult human heart pathology than in the ameroid-induced
MI porcine model, as most patients with MI already have endothelial dysfunction in the microvasculature to some extent. Such patients are usually elderly and have multiple comorbidities, including diabetes, hypertension, and dyslipidemia, which may also enhance the effect on microvascular function and remodeling process. Second, although the porcine model has been reported to show less 
A

B

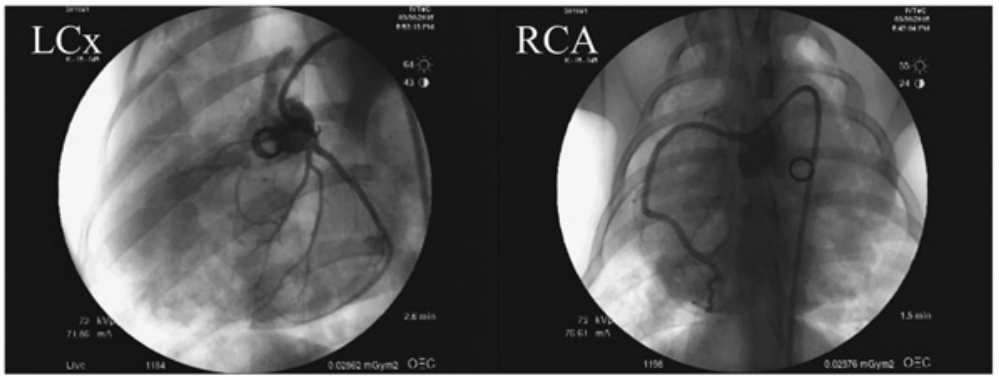

C

IMR

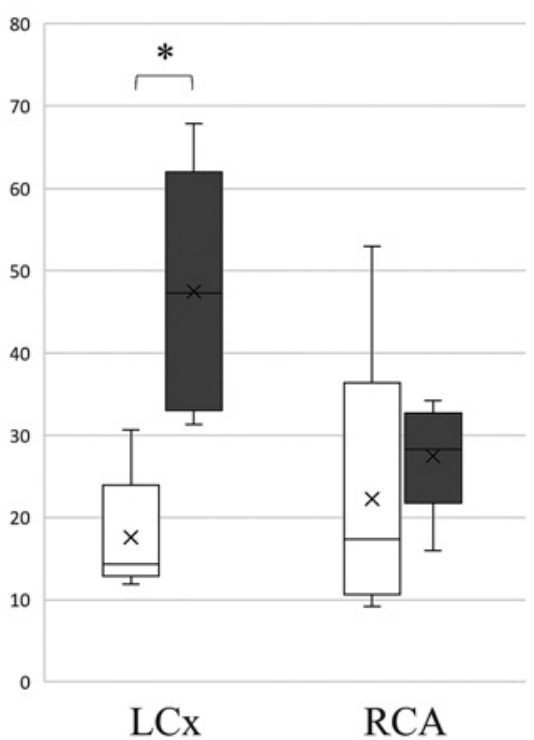

CFR

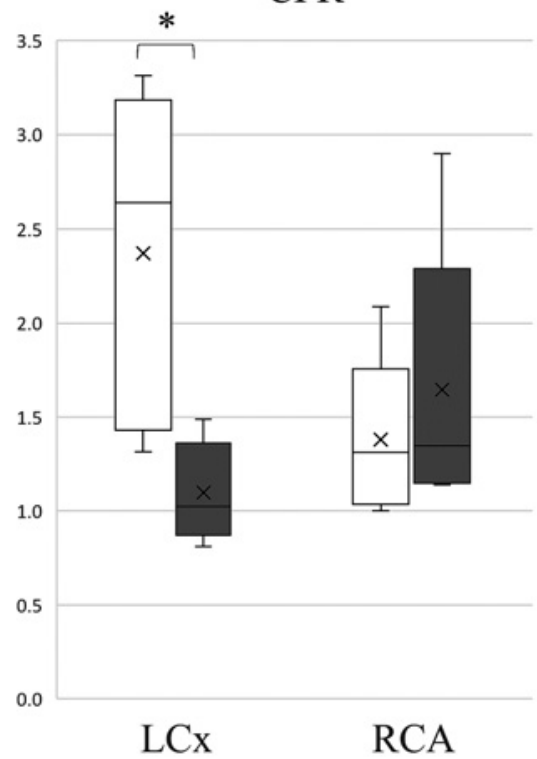

\section{Control $\square 8$ weeks}

Figure 6. Representative coronary angiogram of the LCx (A) and RCA (B). C: IMR and CFR in the LCx and RCA territories in the controls and at eight weeks $(n=5)$. The upper and lower borders of the box represent the upper and lower quartiles, respectively. The middle horizontal line represents the median. The upper and lower whiskers represent the maximum and minimum values of nonoutliers, respectively. The extra dots represent outliers. ${ }^{*} P<0.05$, as assessed using the MannWhitney $U$ test. LCx indicates left circumflex artery; RCA, right coronary artery; CFR, coronary flow reserve; and IMR, index of microvascular resistance.

collateral artery development than that in human patients, the present model characterizes the myocardial physiology of an avascular region in which no blood is perfused, such as the case of an ischemic myocardium untreatable by percutaneous coronary intervention or coronary artery bypass grafting.

Microvascular dysfunction in the infarct-border region: In previous reports comparing the myocardial physiology between infarcted and noninfarcted regions, it has been demonstrated that microvascular dysfunction develops even in noninfarcted regions. In the present study, we clarified that myocardial microvascular dysfunction does not occur in the remote regions, but it does occur in the infarct-border region; both regions have been previously described as noninfarcted regions. ${ }^{7,20)}$ In addition, regional systolic cardiac wall motion and MBF in the infarctborder region, but not in the remote region, were significantly lower at eight weeks after the ischemic insult compared to that in the controls. Interestingly, the MBF in the border area at eight weeks, but not at four weeks, was significantly smaller than that in the controls, indicating that microvascular dysfunction progressed over the four weeks. We speculated that necrotic tissue derived from the MI or microthrombi-induced vasoconstrictor substances, such as thromboxane A2 or serotonin, ${ }^{21)}$ decreased regional MBF and increased microvascular resistance, leading to the extension of ischemia. We believe that it is important to elucidate the site or time course of myocardial 

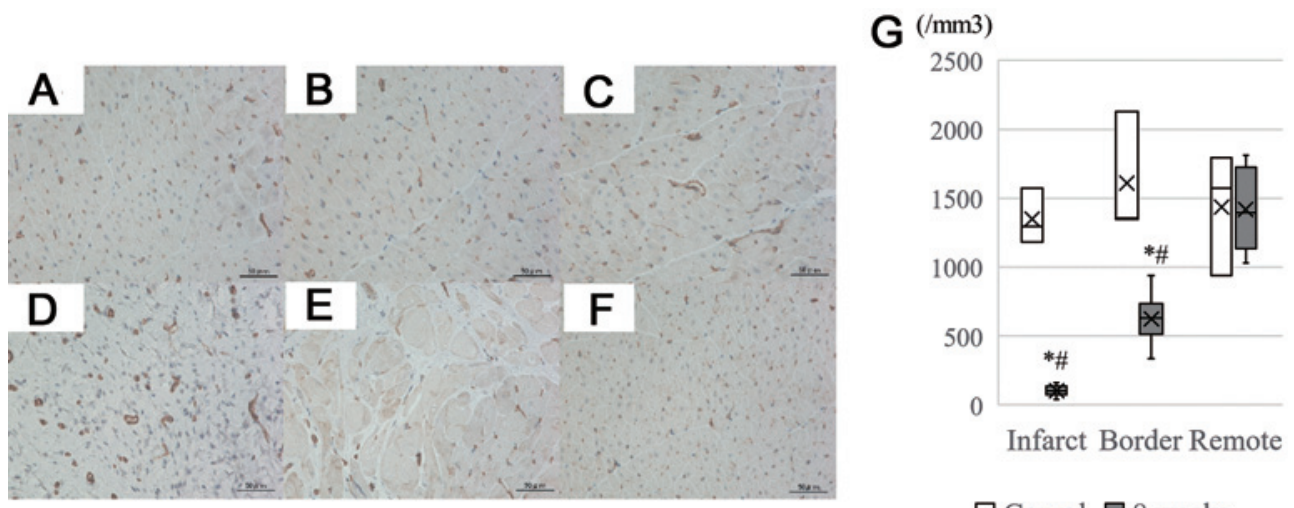

\section{$\square$ Conrol $\square 8$ weeks}
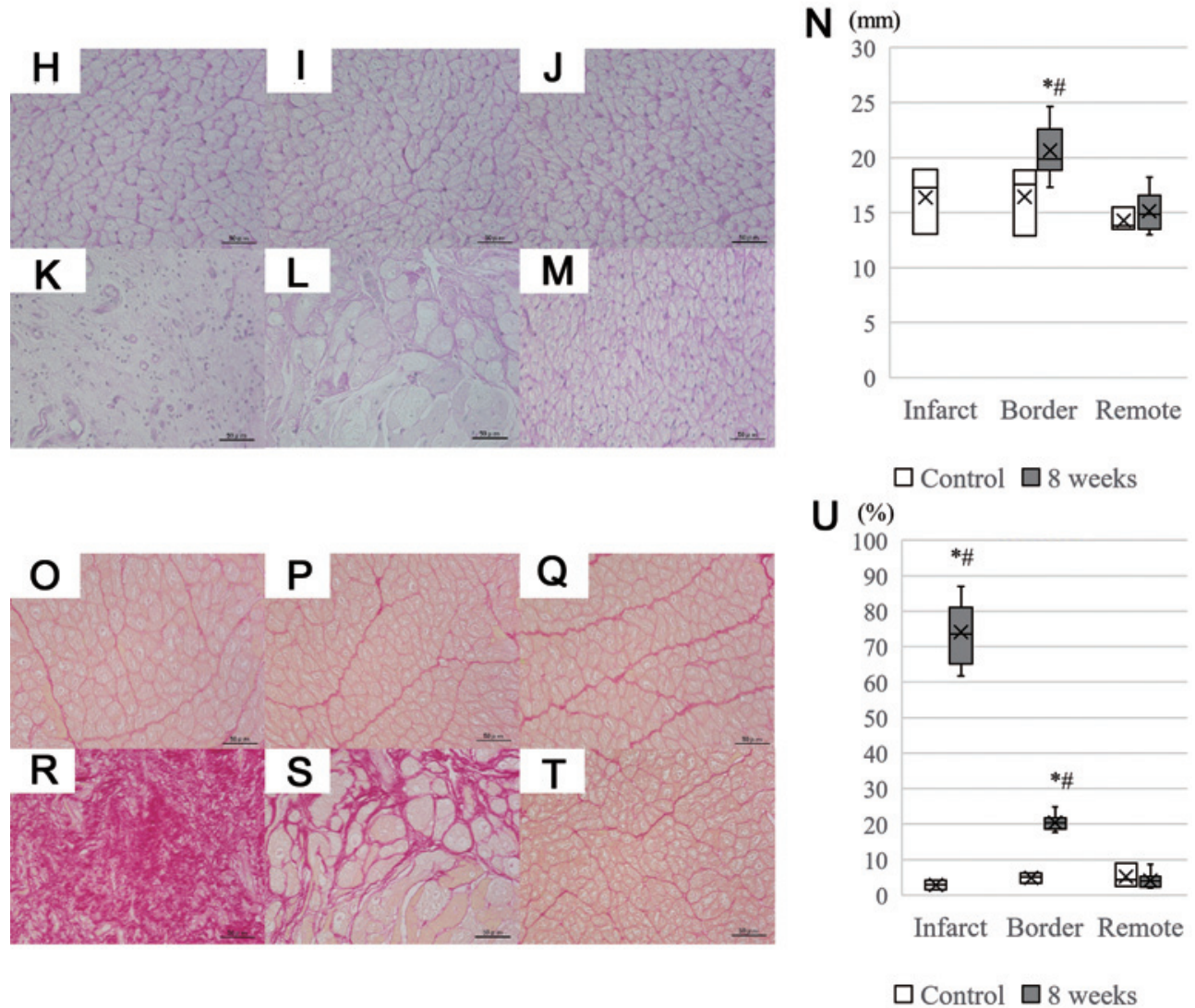

Figure 7. Representative figures of immunostaining labeling for $\mathrm{CD} 31$ in the infarct (A), peri-infarct (B), and remote $(\mathbf{C})$ areas, as well as in the corresponding areas in the normal heart (D-F). The number of CD31-positive capillaries in the infarct, peri-infarct, and remote areas for the normal heart and at eight weeks after LAD occlusion (G). Representative figures of PAS staining in the infarct $(\mathbf{H})$, peri-infarct $(\mathbf{I})$, and remote $(\mathbf{J})$ areas, as well as in the corresponding areas in the normal heart (K-M). The myocyte size in the infarct, peri-infarct, and remote areas for the normal heart and at eight weeks after LAD occlusion $(\mathbf{N})$. Representative figures of fibrosis in the infarct $(\mathbf{O})$, peri-infarct $(\mathbf{P})$, and remote $(\mathbf{Q})$ areas, as well as in the corresponding areas in the normal heart $(\mathbf{R}-\mathbf{T})$. The myocyte size in the infarct, peri-infarct, and remote areas for the normal heart and at eight weeks after LAD occlusion (U) (normal, $n=3$; eight weeks, $n=5$ ). The upper and lower borders of the box represent the upper and lower quartiles, respectively. The middle horizontal line represents the median. The upper and lower whiskers represent the maximum and minimum values of nonoutliers, respectively. The extra dots represent outliers. ${ }^{*} P<0.05$ compared to normal heart, ${ }^{\#} P<0.05$ compared to the value of the remote region at eight weeks, as assessed using Kruskal-Wallis analysis and the MannWhitney $U$ test. LAD indicates left anterior descending artery.

physiological changes in the untreated MI heart, as the findings may provide keys to preventing the extension of myocardial ischemia or improving regenerative therapy, thereby contributing to global cardiac functional recovery. The effect of artery territory: We demonstrated that microvascular dysfunction could develop not only in the 


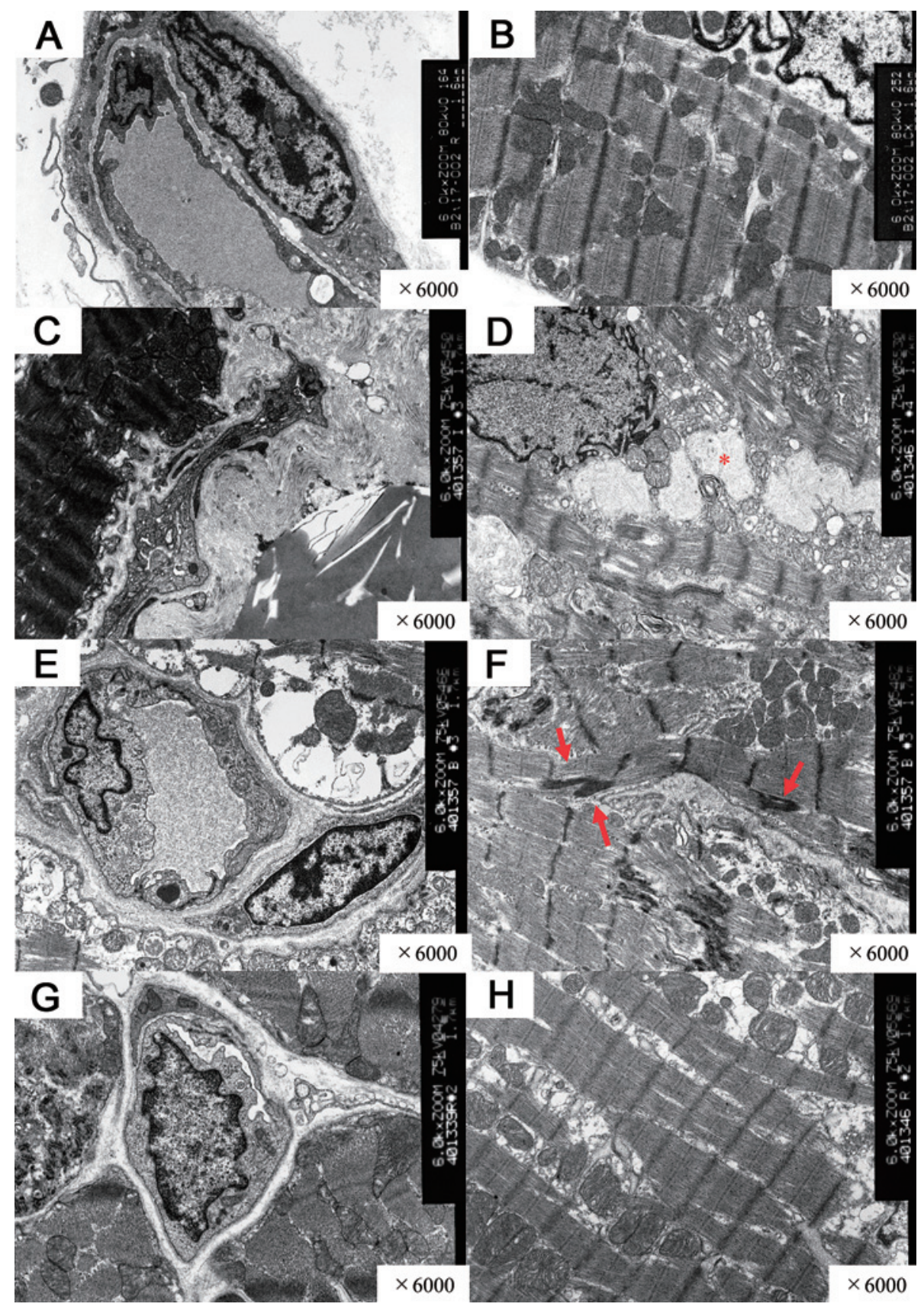

Figure 8. Representative TEM findings of the capillaries and myofibrils in the normal heart $(\mathbf{A}, \mathbf{B})$ and in the infarct $(\mathbf{C}, \mathbf{D})$, border $(\mathbf{E}, \mathbf{F})$, and remote $(\mathbf{G}, \mathbf{H})$ areas, respectively. *Vacuolar degeneration of myofibrils; red arrow: disarrayed Z-bands.

LCx territory, but also in the RCA territory, which is evidently apart from the ischemic insult. Regional systolic cardiac wall motion at four and eight weeks and MBF at eight weeks after the ischemic insult were significantly smaller than in the controls for the RCA territory. In contrast, these values in the remote region were comparable to those in the controls. Thus, the infarct-border region of the RCA territory was also damaged, but not as seriously as the LCx territory. However, we failed to show significant differences in the IMR and CFR in the RCA territory of the ischemic heart compared to that in the controls. This result could be explained by the fact that the LAD directly affects the area of the circumflex territory rather than the right coronary territory as, at the microvascular level, both coronary arteries are connected to form a microvascular network that supplies the LV. Therefore, microvascular dysfunction of the LAD artery mainly affects the circumflex territory. The tendency toward higher IMR and lower CFR in the RCA territory likely depends on the severity of the ischemic insult. 
Limitations: The present study had a few limitations. First, the sample size was small, possibly resulting in insufficient statistical power. Although some intergroup comparison data might be insignificant owing to type 2 statistical errors, those with results still showed clear timely responses of linear and cubic association between controls and four and eight weeks with statistical significance. Therefore, the core outcomes and conclusions remain valid. Second, we did not evaluate the infarct size, myocardial necrotic markers, or myocardial apoptosis in this study. The MI size would have a great influence on LV remodeling. ${ }^{22)}$ Third, the variance in the range of defined myocardial areas among individuals of the same species could have affected the results of the present imaging study. However, as we assessed anomalies using a coronary angiogram and found none, the differences can be minor. Finally, no molecular biological assessments were performed. Clarification of inflammatory or fibrotic processes in the infarct-border region would contribute to a better understanding of the progressive deterioration in the regional myocardial function.

\section{Conclusions}

Clinically, the present results suggest the importance of assessing the microvascular function of the infarct and peri-infarct areas, which would dynamically change after the onset of MI. Additional treatments to stabilize or enhance the microvascular function in the peri-infarct area would contribute to improved clinical outcomes in terms of ST elevation in MI. In conclusion, anterior MI by LAD occlusion causes coronary microvascular dysfunction in the peri-infarct area and is associated with a progressive reduction in the MBF and regional wall motion.

\section{Acknowledgments}

We thank Nao Mukouyama and Masashi Azuma of Kiwa Laboratory Animals Co. Ltd.; Yasuo Hara, Masayuki Maniwa, and colleagues of the Intervention Technical Center in Kobe; and the staff of the PET Molecular Imaging Center in Osaka University Graduate School of Medicine, for their excellent technical assistance.

\section{Disclosure}

Conflicts of interest: The authors declare that there is no conflict of interest.

\section{References}

1. Kawamoto T, Yoshida K, Akasaka T, et al. Can coronary blood flow velocity pattern after primary percutaneous transluminal coronary angiography predict recovery of regional left ventricular function in patients with acute myocardial infarction? Circulation 1999; 100: 339-45.

2. Takahashi T, Hiasa Y, Ohara Y, et al. Usefulness of coronary flow reserve immediately after primary coronary angioplasty for acute myocardial infarction in predicting long-term adverse cardiac events. Am J Cardiol 2007; 100: 806-11.

3. Kim DW, Her SH, Park MW, et al. Impact of postprocedural
TIMI flow on long-term clinical outcomes in patients with acute myocardial infarction. Int Heart J 2017; 58: 674-85.

4. Menees DS, Peterson ED, Wang Y, et al. Door-to-balloon time and mortality among patients undergoing primary PCI. N Engl J Med 2013; 369: 901-9.

5. Camici PG, Crea F. Coronary microvascular dysfunction. N Engl J Med 2007; 356: 830-40.

6. Paulus WJ, Tschope C. A novel paradigm for heart failure with preserved ejection fraction: comorbidities drive myocardial dysfunction and remodeling through coronary microvascular endothelial inflammation. J Am Coll Cardiol 2013; 62: 263-71.

7. van de Hoef TP, Bax M, Meuwissen M, et al. Impact of coronary microvascular function on long-term cardiac mortality in patients with acute ST-segment-elevation myocardial infarction. Circ Cardiovasc Interv 2013; 6: 207-15.

8. Teramoto N, Koshino K, Yokoyama I, et al. Experimental pig model of old myocardial infarction with long survival leading to chronic left ventricular dysfunction and remodeling as evaluated by PET. J Nucl Med 2011; 52: 761-8.

9. Miyagawa S, Sawa Y, Sakakida S, et al. Tissue cardiomyoplasty using bioengineered contractile cardiomyocyte sheets to repair damaged myocardium: their integration with recipient myocardium. Transplantation 2005; 80: 1586-95.

10. Kraitchman DL, Heldman AW, Atalar E, et al. In vivo magnetic resonance imaging of mesenchymal stem cells in myocardial infarction. Circulation 2003; 107: 2290-3.

11. Cerqueira MD, Weissman NJ, Dilsizian V, et al. Standardized myocardial segmentation and nomenclature for tomographic imaging of the heart. A statement for healthcare professionals from the Cardiac Imaging Committee of the Council on Clinical Cardiology of the American Heart Association. Circulation 2002; 105: 539-42.

12. Obokata M, Nagata Y, Wu VC, et al. Direct comparison of cardiac magnetic resonance feature tracking and 2D/3D echocardiography speckle tracking for evaluation of global left ventricular strain. Eur Heart J Cardiovasc Imaging 2015; 17: 52532 .

13. DeGrado TR, Hanson MW, Turkington TG, et al. Estimation of myocardial blood flow for longitudinal studies with $13 \mathrm{~N}$-labeled ammonia and positron emission tomography. J Nucl Cardiol 1996; 3: 494-507.

14. Fearon WF, Balsam LB, Farouque HM, et al. Novel index for invasively assessing the coronary microcirculation. Circulation 2003; 107: 3129-32.

15. Ozawa H, Miyagawa S, Fukushima S, et al. Sirtuin1 regulates the stem cell therapeutic effects on regenerative capability for treating severe heart failure in a juvenile animal model. Ann Thorac Surg 2016; 102: 803-12.

16. Mancuso MR, Davis R, Norberg SM, et al. Rapid vascular regrowth in tumors after reversal of VEGF inhibition. J Clin Invest 2006; 116: 2610-21.

17. Wang Z, Ding J, Luo X, et al. Effect of allopurinol on myocardial energy metabolism in chronic heart failure rats after myocardial infarct. Int Heart J 2016; 57: 753-9.

18. Shudo Y, Miyagawa S, Fukushima S, et al. Novel regenerative therapy using cell-sheet covered with omentum flap delivers a huge number of cells in a porcine myocardial infarction model. J Thorac Cardiovasc Surg 2011; 142: 1188-96.

19. Kawamura M, Miyagawa S, Fukushima S, et al. Enhanced survival of transplanted human induced pluripotent stem cellderived cardiomyocytes by the combination of cell sheets with the pedicled omental flap technique in a porcine heart. Circulation 2013; 128: S87-94.

20. Uren NG, Crake T, Lefroy DC, de Silva R, Davies GJ, Maseri A. Reduced coronary vasodilator function in infarcted and normal myocardium after myocardial infarction. $\mathrm{N}$ Engl J Med 1994; 331: 222-7.

21. McFadden EP, Clarke JG, Davies GJ, Kaski JC, Haider AW, Maseri A. Effect of intracoronary serotonin on coronary vessels in patients with stable angina and patients with variant angina. 
N Engl J Med 1991; 324: 648-54.

coronary blood flow. Compr Physiol 2017; 7: 321-82. 